\title{
Local Anaesthesia in Plastic Surgery Procedures
}

\author{
Ms.Rupali Sawant \\ University Hospital of South Manchester,UK.
}

\begin{abstract}
It is essential to have adequate anaesthesia, to carry out any surgical procedure. Anaesthesia is loss of sensation. Anaesthesia could be local, regional or general. General anaesthesia is the induction of a state of unconsciousness with the absence of pain sensation over the entire body, through the administration of anaesthetic drugs. Local anaesthesia is reversible loss of sensation over a small part of body. Regional anaesthesia typically affects a larger area than local anaesthesia. Many plastic surgery procedures involving smaller part of body, including primary suturing or reconstruction of skin defects by skin grafting, excision of skin lesions, hand surgeries are preferred to be done under local anaesthesia. Precautions should be taken to avoid toxicity of anaesthetic as well as administer it safely.
\end{abstract}

\section{Introduction}

Local anaesthesia (LA) is application of an anaesthetic drug to a specific area of the body, as opposed to the entire body and brain as occurs during general anaesthesia (GA). Local anaesthesia is reversible loss of sensation over a small part of body.[1] For procedures which involve smaller area of body, use of local anaesthesia has more advantages over general anaesthesia.

\section{Methods}

Application of local anaesthesia-

- Proper assessment of site of surgery and anaesthesia by surgeon.

- Choice of anaesthesia is decided by operating surgeon.

- In theatre monitor patients' BP and HR, Equipment for CPR needs to be kept ready.

- The dose of anaesthetic used should be less than the toxic dose.

Epinephrine (1:100,000 to 1:200,000 dilution) may be added to anaesthetic solutions in local infiltrations and field blocks to enhance vasoconstriction, which decreases systemic absorption and prolongs the duration of anaesthesia.[2,3]

Table 1: Anaesthetic agents used for LA[2]:

\begin{tabular}{|l|l|}
\hline Aminoesters & Aminoamides \\
\hline Chloroprocaine & Lignocaine \\
\hline Cocaine & Bupivacaine \\
\hline Procaine & Mepivacaine \\
\hline Tetracaine & Prilocaine \\
\hline Benzocaine & Ropivacaine \\
\hline Piperocaine & \\
\hline
\end{tabular}

Table 2: Toxic doses [5-8]-

\begin{tabular}{|l|l|}
\hline Local anaesthetic & Maximum dose(mg/Kg) \\
\hline Lidocaine & $3 \mathrm{mg} / \mathrm{Kg}$ \\
\hline Lidocaine with Adrenaline(1:200000) & $7 \mathrm{mg} / \mathrm{Kg}$ \\
\hline Prilocaine & $6 \mathrm{mg} / \mathrm{Kg}$ \\
\hline Ropivacaine & $3 \mathrm{mg} / \mathrm{Kg}$ \\
\hline Bupivacaine & $2 \mathrm{mg} / \mathrm{Kg}$ \\
\hline (Levo)Bupivacaine & $2 \mathrm{mg} / \mathrm{Kg}$ \\
\hline
\end{tabular}

Types of local anaesthesia:

1. Local application- For procedures on intact skin in children, one commonly used topical agent is EMLA cream (theraputectic mixture of local anaesthetics), a combination of 2.5 percent lidocaine and 2.5 percent prilocaine. EMLA has proven benefits but is limited by its delayed onset of action (one to two hours after application). $[3,4]$

2. Infiltration- This type of anaesthesia is used for small lacerations and skin biopsies. The anaesthetic solution is infiltrated to the deep dermis, where the sensory plexus supplying the skin begins to branch. 
3. Field block- In the field block, anaesthetic is infiltrated to the subcutaneous area surrounding the operative field. Few common examples are-

Forehead block: The forehead is supplied by the supraorbital and supratrochlear nerves. The supraorbital nerve emerges from the supraorbital foramen, which can be palpated along the upper border of the orbit, approximately $2.5 \mathrm{~cm}$ lateral to the midline of the face. The supratrochlear nerve exits along the upper border of the orbit, approximately $1 \mathrm{~cm}$ medial to the supraorbital foramen. The local anaesthetic is injected along superior orbital margin near the nerve, injection directly into the foramen should be avoided to decrease the risk of nerve damage. [8]

Infraorbital Nerve Block: The nerve exits from the infraorbital foramen, which is located just inferior to the orbit slightly nasal to an imaginary line drawn through the middle of the infraorbital rim. For the nerve block, the needle is inserted approximately $1 \mathrm{~cm}$ inferior to the infraorbital foramen and advanced upward toward the foramen. The needle is directed upward and laterally to avoid passing through the foramen into the orbit.

Ear Block: The ear is supplied by the greater auricular, lesser occipital, and auriculotemporal nerves, and the mastoid branches of the lesser occipital nerve. [8] A field block is required for anaesthesia. Local anaesthetic is injected in post and preaural region superiorly and inferiorly.

Lip Block: Upper lips are anesthetized from two either side of the angle of the mouth. An anaesthetic is injected along two lines in the direction of the nasal alae. For lower lip anaesthesia, the needle isinjected at the midpoint of the chin and soft tissues are infiltrated obliquely upward toward the angle of the mouth. [8]

4. Nerve block- Nerve block is achieved by infiltrating the anaesthetic over the region where the particular nerve is situated. Nerve blocks involve the injection of an anesthetic into the area around a nerve that supplies a particular region of the body, preventing the nerve from carrying nerve impulses to the brain. Some common examples are-

\section{Mental Nerve Block}

The mental nerve supplies the anterior aspect of the mandible. It emerges from the mental foramen, which lies between the upper and lower borders of the mandible, and is located on the continuation of an imaginary line from the supraorbital foramen through the pupil and the infraorbital foramen. [8] The needle is injected about $1.5 \mathrm{~cm}$ posterior and lateral to the foramen and directed toward the foramen.

\section{Median Nerve Block}

The median nerve provides sensations to the radial aspect of the palm, palmar surface of the thumb, index finger, middle finger, radial half of the ring finger, and the nail beds of the same digits.[9] The nerve enters the hand between the Flexor carpi radialis and Palmaris longus tendons beneath the flexor retinaculum. Both tendons are identified by asking the patient to oppose the thumb and the fifth digit. [10] The anaesthetic is injected by keeping the needle angled at 45 degrees and enter between the tendons at the level of the proximal wrist crease. As the needle passes through the flexor retinaculum, a loss of resistance is felt, marking the point at which the injection should be made. If there is symptom of paresthesia, the needle should be withdrawn slightly to avoid nerve fibre damage or intraneural injection.

A superficial palmar branch supplying the skin over the thenar area can be blocked by subcutaneous injection of anaesthetic above the retinaculum.[11]

\section{Radial Nerve Block}

The superficial branch of the radial nerve supplies the dorsum of the hand and first three fingers proximal to the distal interphalangeal joint. [12] First infiltration, just lateral to the radial artery at the proximal wrist crease. The needle is then redirected and advanced subcutaneously across the proximal border of the snuffbox toward the middle of the dorsal wrist. Several needle entry sites may be necessary to follow the curve of the radial aspect of the wrist and adequately cover the entire area.

\section{Ulnar Nerve Block}

The ulnar nerve divides into palmar and dorsal branches at the proximal flexor crease of the wrist, supplying the ulnar aspect of the palmar and dorsal surface of the hand and fifth finger, and ulnar half of the fourth finger. Injection of anaesthetic 1 to $2 \mathrm{~cm}$ deep between the Flexor carpi ulnaris tendon and the ulnar artery blocks the palmar branch of the ulnar nerve. The dorsal ulnar branch is blocked by subcutaneous infiltration of anaesthetic distal to the ulnar styloid process. Both branches of the ulnar nerve also can be blocked at the elbow. With the elbow flexed at 90 degrees, the anaesthetic is infiltrated into the skin between the olecranon and the medial epicondyle. [13] The potential for nerve damage is higher with this procedure because the nerve can be trapped between local anaesthetic and the bone, causing nerve ischemia.[14]

\section{Digital Nerve Block}

A web space block - The needle is inserted from the dorsal aspect of the web space and advanced until the tip tents the palmar skin. Anaesthetic is administered along the side of the digit as the needle is withdrawn. 
Care should be taken not to infiltrate circumferentially, which can lead to vascular compromise. Epinephrine should be used with caution in digital blocks.

Transthecal block- The hand is positioned palm upwards and the injection site is prepared with isopropyl alcohol. The flexor tendon is palpated on the palmar surface over and just proximal to the metacarpophalangeal joint. It may be made more obvious by gentle flexion of the appropriate finger. A 2-mm syringe with a 25 gauge needle attatched is used to introduce the local anaesthesic (lignocaine 1\%). The skin is penetrated at an angle of 45 degrees at the level of the distal skin crease of the palm just proximal to the metacarpo- phalangeal joint, and the needle is advanced to the level of the flexor tendon sheath. If the sheath has been entered local anaesthesia should flow freely when gentle pressure is applied to the plunger. In 1990, Chiu [15] proposed a single volar transthecal injection that uses the flexor sheath for infusion of the anesthetic solution. His technique was simplified by Whetzel et al [16], who use the proximal digital crease as a visual landmark for injection.

Subcutaneous block- Harbison [17] proposed a subcutaneous single injection digital block that does not violate the flexor tendon sheath but is still very efficient and straightforward to provide finger anaesthesia. The only disadvantage reported by Harbison is that the dorsal digital nerves are not always anesthetized. The subcutaneous digital block described by Harbison allows for the treatment of all conditions along the volar aspect of the finger and on the dorsal aspect of the distal and middle phalanxes without the need to enter the flexor tendon sheath. Associated risks are, therefore, avoided and, thus, the subcutaneous digital block should be preferred to the transthecal techniques to avoid flexor sheath injury.[18]

\section{Discussion}

\section{Local anaesthesia:}

Mechanism of action- Anaesthetic agent blocks nerve conduction by reversibly binding and inhibiting sodium channels. Local anaesthetics (LAs) are classified into esters and amides. Most ester LAs are metabolized by pseudo-cholinesterase, while amide LAs are metabolized in the liver. In patients with liver failure, esters are chosen for local anaesthesia. Choice of anaesthetic and type of block is surgeon's decision. Anesthetics may be administered with another drug, such as epinephrine (adrenaline), which decreases bleeding, and sodium bicarbonate to decrease the acidity of a drug so that it will work faster. [1]

Local anaesthesia is a much safer alternative to general anaesthesia because:

- It does not put the body under stress in the same way as GA.

- Most importantly, fewer drugs are needed so recovery from the procedure is faster.

- Patients with surgeries under LA can be discharged home early, reducing the hospital stay.

- Required procedures can be done under LA in patients with multiple co-morbidities.

- Expectant result of the surgery may be assessed.

Limitations of local anaesthesia/Nerve block [19]:

- Allergy to anaesthetic agent

- Infection at injection site

- Poor patient compliance

- Surgeons lack of familiarity with nerve block.

- Coagulopathy

Adverse effects:

Side effects of regional or local anesthetics vary depending on the type of anaesthetic used and the way it is administered [8]:

- Localized prolonged anaesthesia or paresthesia due to infection, hematoma, excessive fluid pressure in a confined cavity, and severing of nerves \& support tissue during injection.

- Systemic reactions such as depressed CNS syndrome,allergic reaction, vasovagal episode, and cyanosis due to local anaesthetic toxicity.

- Lack of anaesthetic effect due to infectious pus such as an abscess.

\section{Toxicity of anaesthetics causes:}

CNS: Light headedness, tinnitus, metallic taste, visual disturbances, numbness of the tongue and lips (rarely progressing to muscle twitching), loss of consciousness, seizures and coma.

Cardiovascular: Decreased ventricular contractility, decreased conduction and loss of vasomotor tone. Systemic toxicity is rare with regional anaesthesia and can be prevented by using the smallest dose possible and aspirating before the injection. Nerve blocks can provide adequate anaesthesia in procedures on small parts of the face, hand and feet. Field block also may be considered when operating on the ear or lips. Care should be taken to avoid compromising blood supply, nerve injury or cause compartment syndrome. Plan and choose type of anaesthesia according to the anatomy of surgical site. 


\section{Conclusion}

Local anaesthesia causes less morbidity and reduces duration of hospital stay. It could be used more efficiently by:

- Use of narrow calibre needle

- Very slow administration of anaesthetic

- Avoiding injecting cold medication

- Aspiration to confirm needle is not in a vessel to avoid intravascular administration.

- Administration of dose less than the maximum dose to avoid toxicity.

- To be aware of anatomical position of nerves

- Avoid direct injury to nerves

\section{References}

[1]. Medical-dictionary.thefreedictionary.com.Gale Encyclopedia of Medicine.

[2]. Orlinsky M, Dean E. Local and topical anesthesia.In: Robert JR, Hedges JR. Clinical procedures in emergency medicine, $3 \mathrm{~d}$ ed. Philadelphia: Saunders, 1998:454-73.

[3]. Huang W, Vidimos A. Topical anesthetics in dermatology.J Am AcadDermatol 2000;43:286-98.

[4]. Sweitzer BJ, Pilla M, Hurford WE, Local anesthetics. In: Massachusetts General Hospital. Department of Anesthesia. Clinical anesthesia procedures of the Massachusetts General Hospital, 5th ed. Philadelphia:Lippincott-Raven, 1998:233-4

[5]. Peck T, Hill S, Williams M (2008). Pharmacology for Anaesthesia and Intensive Care. 3rd ed. Cambridge: Cambridge University Press.

[6]. Smith A, ScartE,Sasada M (2011). Drugs in Anaesthesia and Intensive Care. 4th ed Oxford: Oxford University Press.

[7]. British National Formulary. September 2014: National Institute of Clinical Excellence (NICE).

[8]. Gohar A. Salam, Regional Anesthesia for Office Procedures: Part I. Head and Neck Surgeries. Am Fam Physician 2004;69(3):58590.

[9]. Melone CP Jr, Isani A. Anesthesia for hand injuries.Emerg Med Clin North Am 1985;3:235-43.

[10]. Raggi RP. Balanced regional anesthesia for hand surgery. Orthop Clin North Am 1986;17:473-82.

[11]. Wedel DJ. Nerve blocks. In: Miller RD, Cucchiara RF. Anesthesia, 5th ed. Philadelphia: Churchill Livingstone, 2000:1520-48.

[12]. Ferrera PC, Chandler R. Anesthesia in the emergency setting: Part I. Hand and foot injuries. Am Fam Physician 1994;50:569-73.

[13]. Smith DW, Peterson MR, DeBerard SC. Regional anesthesia. Nerve blocks of the extremities and face. Postgrad Med 1999;106:6973,77-8

[14]. Gohar A. Salam, Regional Anesthesia for Office Procedures:Part II. Extremity and Inguinal Area Surgeries. Am Fam Physician 2004;69(4):896-900.

[15]. Chiu DTW. Transthecal digital block: Flexor tendon sheath used for anesthetic infusion. J Hand Surg (Am) 1990;15:471-7.

[16]. Whetzel TP, Mabourakh S, Barkhordar R. Modified transthecal digital block. J Hand Surg (Am) 1997; 22:361-3.

[17]. Harbison S. Transthecal digital block: Flexor tendon sheath used for anesthetic infusion. J Hand Surg (Am) 1991;16:957.

[18]. JP Brutus, A Nikolis, Y Baeten, N Chahidi, et al. Reducing patient discomfort during digital blockade: The subcutaneous single injection digital block - A simple, safe and fast procedure. Can J Plast Surg. 2003 Spring; 11(1): 33-35.

[19]. Barash PG, Cullen BF, Stoeling RK. Peripheral nerveblockade. In: Handbook of clinical anesthesia, 2ded. Philadelphia: Lippincott, 1993:238-55. 